\title{
Android Operated Robotic Arm
}

\author{
Zain Anwar Ali ${ }^{1{ }^{* *}, \text { M.Tanveer }}{ }^{2}$, Habib Shaukat ${ }^{2}$, Saad Anwar ${ }^{3}$ \\ ${ }^{1}$ College of Automation Engineering, Nanjing University of Aeronautics \& Astronautics, China \\ ${ }^{2}$ Depart. of Electronic Engineering, Sir Syed University of Engineering \& Technology, Pakistan \\ ${ }^{3}$ Depart. of Computer Engineering, Sir Syed University of Engineering \& Technology, Pakistan \\ *Corresponding Author: zainanwar86@hotmail.com
}

Copyright @ 2014 Horizon Research Publishing All rights reserved.

\begin{abstract}
As the research project name suggest, the android operated robot arm is designed so as it can perform the activities of a human arm by the instructions given through android device (android app). A signal is generated when specific buttons on the android application are pressed which allows microcontroller to make the arm move according to the predefined program. Here Android application being the command center of the robotic arm as it commands the arm to move or grab specific things as the instruction is transferred to the arm through android JAVA and microcontroller language. At the receiver the signal is provided to the microcontroller which does several manipulations in this data using different scaling factors through programming, which controls the direction and speed of the motors that is responsible for the motion of the robotic arm.
\end{abstract}

Keywords Android Robot, Robotic Arm using Android, Modern Robot

\section{Introduction}

The summarize detail description of each segment and its working. In this research is partitioned in several stages covering from acquiring data to its implementation. Several calculations and design circuits have been considered. The individual segments are described in detailed below. The few parts that a robotic arm must possess are:

\section{Glove}

This section is responsible for capturing and grabbing movements of fingers and wrist. It is designed like a normal wearing glove made of leather. For every movement in the finger of robotic arm, there is a specific button described in the android app. The second part, the wrist motion, has also been captured using the same procedure. Similarly in human wrist there are two side motions up down and right left, to avoid complexity we have only sensed the up and down motion by using servo motor which is moved by the instruction given through applications at the predefined angles [10].

\section{Elbow Pad}

The human elbow works in conjunction with the shoulder that is as a lifting or rotating joint as with our case we have use the elbow joint as a lifting joint because we rotate the arm using shoulder. Therefore we use two buttons in the application just to rotate the motor upwards or downwards.

\section{Shoulder Pad}

'The shoulder of human arm is itself as a ball joint in its formation responsible for revolving, rotating and lifting. As it is the backbone of the arm and carries the load of entire arm (Elbow and wrist joints) we have two buttons in the application for the movement of the shoulder motor upwards or downwards. The receiver circuit is attached with the microcontroller to which all the circuitry is attached.

The receiver circuit thus used with the microcontroller is IOIO board for Android which accepts commands from the microcontroller and transmits signals for the microcontroller. These signals are further processed by microcontroller (depending on which pin of the microcontroller the signal is received) and performs the pre defined actions defined in the microcontroller program [3].

\section{Andoird Board / Panel}

The IOIO (distinct as "yo-yo") is a panel specifically planned to effort with your Machine 1.5 and later device. The panel arrange for robust communication to an Android device via a Bluetooth or USB link and is entirely well-regulated from within an Android request via guileless and instinctive Java API - no fixed software development or outward programmer will ever be required [3]. The yo-yo panel or board holds only MCU that work as a USB crowd and infers guidelines from an Android application [9]. In accumulation, the yo-yo can network with marginal devices in the same way as most MCUs [1]. Arithmetical Input/Output, PWM, Similarity Input, I2C, SPI, and UART mechanism be able to all be cast-off with the yo-yo board. Encryption to switch these interfaces is transcribed in very 
similar way as you compose an Android application with the support of a modest to usage the application-level archive [1]. Beside these, it will be able to combine the splendid calculating power, Bluetooth \& Internet connectivity, touch panel screen, and a lot of sensors with this Android device with the capability to simply add marginal devices to communicate with the outer world. Further-more using the IOIO organizer did not require any software \& hardware modifications to your Android devices.

\section{AT 89C51 Microcontroller}

The microcontroller executes all the controlling responsibilities. Atmel 89C51 microcontroller is operating at $12 \mathrm{MHz}$ of frequency. The yo-yo panel board transmit data on the pins of the 89C51 microcontroller. The controller then identifies at which pin the sign has been sent and then it deals the motors of the arm accordingly. The microcontroller is also capable of serial transmission; the above frame is serially transmitted to the transmitter section. The microcontroller is used in 8 bit UART mode with 1 start bit, 8 data and 1 stop bit at $19.2 \mathrm{kbps}$ baud rate [8].

\section{Wireless Transmission and Reception}

IOIO board communicates with your Android device over Bluetooth connection. This is achieved simply by attaching a standard USB Bluetooth dongle to the IOIO board [2].

a. Connection

The complete procedure of connection is defined as above in the following steps.

- $\quad$ By using IOIO v1 panels, to plug the dongle into the USB jack on the IOIO board [4].

- On IOIO-OTG panels, use the delivered USB male-micro (connector)-A-to-womanly-A (connector) adapter cable [2].

- Make definite control of preventive potentiometer is all the way clockwise.

\section{b. Pairing}

To command for a request to be talented to recognize your yo-yo panels within array, the primary need to be "paired" with the Android method [6]. Pairing is a one-time process done per dongle. Once paired, the Android device is going to recollect the definite dongle and will not need pairing again and again.

Following the steps of procedure of pairing the IOIO device.

- To provide Power on IOIO board and attach the Bluetooth dongle to it.

- $\quad$ Firstly go to Settings of android device $>$ Secondly Wireless \& networks $>$ after that Bluetooth settings menu. Moreover make sure Bluetooth is activated by checking the top box. Then hit the button "devices for scanning".
- A method called IOIO (xx:xx) is realized, where $\mathrm{xx}: \mathrm{xx}$ are the previous 4 digits of any IOIO address. When additional IOIO is used, every module will have a dissimilar address, generating it possible to distinct between them. The addresses arises from the dongle and not from the IOIO, so if exchange two dongles, the addresses will spirit with the dongles and not with the IOIOs [4].

- $\quad$ Click the IOIO in order to pair. It will be asked for a PIN code. Enter 4545 [5].

- $\quad$ IOIO is now paired, until we manually unpaired it (by long clicking the device from the same screen).

Once the connection has been made and pairing is set up, it's up to the android java to send signals to IOIO board which further transfers the signal to $89 \mathrm{C} 51$ microcontroller [6].

\section{c. Motors and Drive Circuit}

By using stepper motor this means how much steps do the motor have to take. For fingers use a stepper motor but for all other joints we have used DC gear motors, for that the microcontroller uses a time and value relationship to achieve the required task. If the value is shifted by one the DC motor is allowed to run for a certain time period proportional to the new value. Microcontroller rotates a specific code at its port which causes the rotation of stepper motor [7]. The forward and backward direction of the stepper motor depends upon the direction of rotation of code. As it is mentioned above that we have used stepper motor for the fingers and DC gear motors for the wrist, elbow and shoulder joint. We have used a Darlington pair circuit for the stepper and two relay switching system for the DC gear motors [7]. We use Darlington pair for each pole of stepper motor that has a capability to sink high current. Transistor 3904 is triggered by micro controller which then trigger a power transistor TIP125.The driving circuitry for a stepper motor thus consist of 4 pairs of 3940 and TIP 125. This circuit can handle up to $5 \mathrm{~A}$ of current.

\section{Working of System}

A person operates the android device with an established connection to the IOIO board. A signal is generated against each button which represents specific movements. These signals are attend by a specific pic on the IOIO board which is directly connected to the input port of $89 \mathrm{C} 51$ microcontroller [8]. This digital data is fed in a microcontroller, which performs several manipulations in this data using different scaling factors through programming and controls the direction and speed of motors which results in the motion of robotic arm.(Figure 1)

\section{Software Interaction}

These are the software's which were used in this research to deal different aspects of programming and to handle 
hardware section by synchronized each of them.

The Keil U vision and protius these software's used for programming Microcontroller. Secondly U Vision debugger for debugging moreover NSP programmer \&Android Eclipse s also used to complete this research or fulfill our goals.
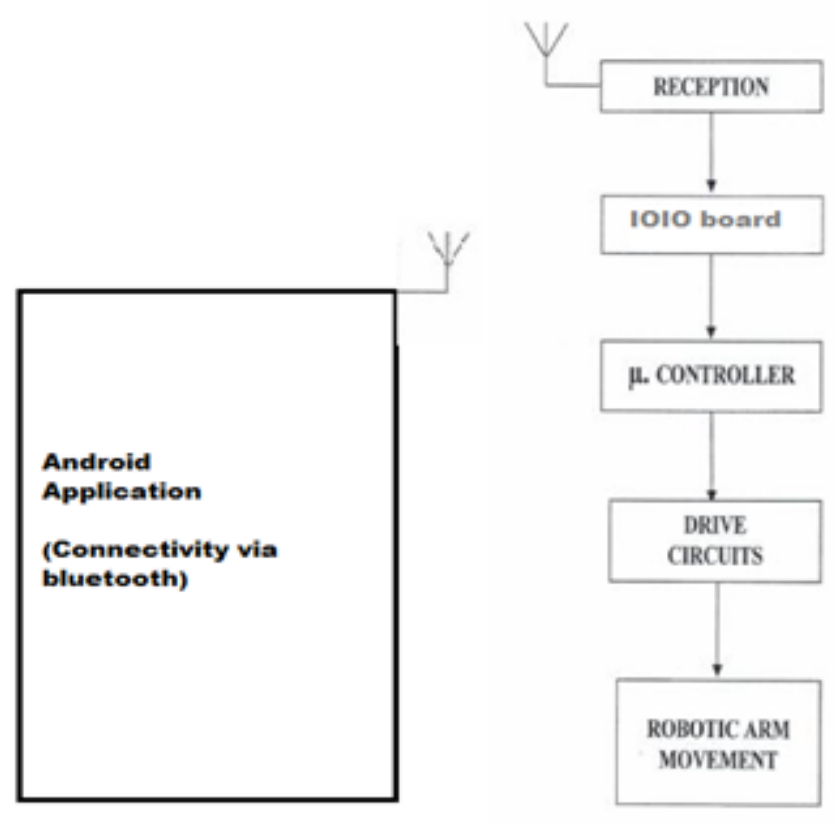

Figure 1. Block Diagram of Complete System Working

\section{Conclusion}

There are many robots in the market but we have designed an arm which can be operated through android device. It was designed keeping in mind that it will facilitate people of different professions. The robotic arm can perform nearly same movements using the stepper and DC motors having a precise control. The use of Aluminum reduces the weight of the arm, making it more durable decreasing the power consumption. Stepper motor increases the precision of the arm. DC motors at the shoulder increase the capability of the arm to lift heavy loads.

Even though this research is quiet in an initial step of improvement, it consumes now proven to flourish in more than a few of its areas. It takes magnificently verified to deliver a robot autonomous and operational-system-liberated application boundary. It consumes also verified to permit for significant two-way statement among the Android organizer and the robot which longed permit a non-expert to relate with and regulate the functionality of a robotic systems. Presently covering in the structure to allow for real-world control of a robotic arm of an Android-permitted smart phone. The completion invention of this research is a presentation which turns on any Android-enabled expedient and permits a non-skilful to automatically link with a variability of engineering robotic apparatus.

\section{Future Scope}

The Android base robotic arm is designed to lift the objects. Right now able to lift the objects having intermediate weight. But in future to extend more weight or heavy weight lifting is required then more innovative tools and materials is requisite. Then it will be able to lift the large containers at the sea-port, in industrial area, military usage or so on.

\section{REFERENCES}

[1] https://www.sparkfun.com/products/retired/10748

[2] https://github.com/ytai/ioio/wiki/IOIO-Over-Bluetooth

[3] http://microcontrollerkits.blogspot.com/2011/10/ioio-board-f or-android-control.html

[4] https://github.com/ytai/ioio/wiki/IOIO-Over-Bluetooth-for-P C

[5] http://www.circuitlake.com/ioio-control-electronics-projectwith-android-phone.html

[6] Z. Mednieks, "Programming Android," O’Reilly Media, ISBN: 1449389697, 2011.

[7] T. Fong, I. Nourbakhsh, and K. Dautenhahn, "A Survey of Socially Interactive Robots," Robotics and Autonomous Systems, vol. 43, pp. 143-166, 2003

[8] http://www.researchgate.net/publication/229045477_Develo pment_of_a_Microcontroller_Based_Robotic_Arm

[9] “Arduino MCU”, - www.arduino.com

[10] "Servomotors \& system design com-ponents", www.bhashaelectronics.com 